\title{
tic\&société
}

Vol. 12, $\mathrm{N}^{\circ} 2$ | 2ème semestre 2018

Numérique et situations de handicap : les enjeux de l'accessibilité

\section{Vers un design accessible, au-delà du design de la forme et de la fonction}

\section{María Inés LAITANO}

\section{(2) OpenEdition \\ Journals}

Édition électronique

URL : http://journals.openedition.org/ticetsociete/2720

DOI : 10.4000/ticetsociete. 2720

\section{Éditeur}

Association ARTIC

\section{Édition imprimée}

Pagination : 103-121

Référence électronique

María Inés LAITANO, « Vers un design accessible, au-delà du design de la forme et de la fonction », tic\&société [En ligne], Vol. 12, № 2 I 2ème semestre 2018, mis en ligne le 10 décembre 2018, consulté le 01 mai 2019. URL : http://journals.openedition.org/ticetsociete/2720 ; DOI : 10.4000/ ticetsociete. 2720 
tic\&société - 12(2), 2018

\section{Vers un design accessible, au-delà du design de la forme et de la fonction}

\section{María Inés LAITANO}

María Inés Laitano est maîtresse de conférences à l'université Paris 13, membre du LabSic et responsable pédagogique du Master 1 Design d'interface multimédia et internet. Ses recherches portent sur la communication par les interfaces numériques, l'accessibilité numérique et le design participatif. Elle a publié des travaux sur l'accessibilité numérique dans la $12^{\text {th }}$ Web for All Conference, l'International Journal of Sociotechnology and Knowledge Development, la Revista Española de Documentación Científica et la Revista Scire, parmi d'autres.

mariaines.laitano@univ-paris13.fr 
Vers un design accessible, au-delà du design de la forme et de la fonction

\section{Vers un design accessible, au-delà du design de la forme et de la fonction}

Résumé: La France, comme plusieurs pays du globe, a développé un cadre légal pour veiller à l'accessibilité numérique de ses services publics de communication, qui repose sur la conformité avec des standards d'accessibilité. La thèse que nous défendons dans cet article est que ces règles sont principalement focalisées sur le design de la forme et de la fonction des interfaces numériques, au détriment du design du symbolique, de l'usage et des relations. En préconisant une interface perceptible, utilisable, compréhensible et robuste, les règles ne regardent que la matérialité et l'utilité de l'artefact à concevoir. Quelques travaux en design accessible ont déjà pointé les limites de ces standards et recommandé un design accessible de l'usage. Nous y adhérons en orientant la réflexion vers un design accessible du symbolique.

Mots-clés : accessibilité numérique, design accessible, design inclusif, WCAG, approche radicale du design.

Abstract: France, like several countries in the world, has developed a legal framework to guarantee the digital accessibility of its public communication services, based on compliance with accessibility standards. This article argues that these accessibility norms are mainly focused on the form and functional design of digital interfaces, to the detriment of symbolic, use and relational design considerations. By recommending a perceptible, usable, comprehensible and robust interface, standards only pay attention to materiality and the usefulness of the artifact to be designed. Some work in accessible design has already pointed out the limits of standards and recommended design for accessible use. We support this position and propose reflections on accessible symbolic design.

Keywords: digital accessibility, accessible design, inclusive design, WCAG, radical approach to design. 
Resumen: Al igual que varios países del mundo, Francia ha desarrollado un marco legal para garantizar la accesibilidad digital de sus servicios públicos de comunicación, basado en la conformidad con los estándares de accesibilidad. La hipótesis que aquí se presenta es que estas reglas de accesibilidad se centran principalmente en el diseño de la forma y de la función de las interfaces digitales, en detrimento del diseño de lo simbólico, del uso y de las relaciones. A pesar de que preconizan una interfaz perceptible, utilizable, comprensible y robusta, las reglas únicamente contemplan la materialidad y la utilidad del artefacto a diseñar. Algunos trabajos en diseño accesible han comenzado a señalar los límites de estos estándares y han recomendado considerar el diseño del uso accesible. El presente texto hace suya esta orientación y reflexiona sobre la necesidad de un diseño accesible de lo simbólico.

Palabras clave: accesibilidad digital, diseño accesible, diseño inclusivo, WCAG Pautas de Accesibilidad para el Contenido Web, enfoque radical del diseño. 
Vers un design accessible, au-delà du design de la forme et de la fonction

\section{Introduction}

L'accessibilité numérique, entendue comme le droit d'accès à la communication numérique au-delà des singularités physiques, psychiques, culturelles, géographiques ou économiques des personnes, joue un rôle fondamental pour l'inclusion sociale. L'opportunité d'accéder à une formation en ligne peut potentiellement améliorer les conditions de vie d'une personne qui rencontre des difficultés pour se déplacer physiquement jusqu'au lieu d'étude. Le volume d'information disponible en format numérique - et susceptible donc d'être lu à partir d'une synthèse vocale - multiplie plusieurs fois le volume d'information existant au format Braille. Les possibilités du numérique ne représentent pas uniquement des facilités de communication pour les situations de handicap - auxquelles la notion d'accessibilité numérique est normalement associée -, mais également vis-à-vis d'autres usagers tels que les personnes âgées, celles faiblement alphabétisées, les internautes novices, les personnes qui ont une connexion internet lente ou qui utilisent des technologies anciennes.

En France, la loi pour une République numérique de 2016 établit que les services de communication en ligne des établissements publics en général et des entreprises dont le chiffre d'affaires excède un certain seuil devraient, dans une période maximale de trois ans, adopter un design qui respecte les règles du Référentiel général d'accessibilité pour les administrations (RGAA) (SGMAP, 2017). L'obligation concerne autant les sites internet que l'intranet, l'extranet, les applications mobiles, les progiciels et le mobilier urbain numérique. Cependant, malgré le cadre législatif qui existe sous différentes formes depuis au moins une dizaine d'années, les barrières d'inaccessibilité persistent (BrailleNet, 2014). Parmi les nombreuses causes, certains auteurs ont souligné la non-pénalisation des éditeurs de logiciels, des opérateurs de services de communication ou des fabricants d'équipements électroniques lorsqu'ils fabriquent des solutions ne respectant pas les règles d'accessibilité (Balin et Gossart, 2015), 
ou encore la méconnaissance de ces règles par les professionnels du Web, doublée par la lacune en matière de formation universitaire (Lespinet-Najib et al., 2015) ainsi que le décalage temporel entre la production de directives politiques et les activités des créateurs de contenus accessibles (Folcher et Lompré, 2012).

Or, même dans les cas exceptionnels où les standards d'accessibilité sont mis en œuvre, leur capacité à rendre la communication numérique complètement accessible reste questionnable. La thèse que nous défendons dans cet article est que les règles d'accessibilité sont principalement focalisées sur le design de la forme et de la fonction des interfaces numériques, au détriment du design du symbolique, de l'usage et des relations. Nous commencerons par présenter en section 2 l'approche interdisciplinaire radicale du design (Zacklad, 2017) qui s'avère à notre sens optimale pour élucider les limitations des standards d'accessibilité actuels. Nous montrerons ensuite en quoi les principes directeurs des règles d'accessibilité prennent en compte la forme et la fonction des interfaces (section 3), pour poursuivre avec des travaux qui pointent les insuffisances des standards et préconisent le design d'une expérience accessible (section 4). Quelques pistes pour un design accessible du symbolique seront enfin proposées en section 5 .

\section{L'approche interdisciplinaire radicale du design}

D'après Zacklad (2017), la science du design est, tout comme les sciences de l'information et de la communication (SIC) ou les sciences de l'éducation, une interdiscipline "à objet » qui s'alimente de différents savoirs disciplinaires plus fondamentaux ou spécialisés pour répondre aux questions qui leur sont posées. Que ceux-ci relèvent des arts appliqués, des sciences de l'ingénieur ou des sciences humaines et sociales, le design

a comme particularité l'utilisation de méthodes sensibles permettant : (1) de spécifier différents éléments de la forme, du fonctionnement, de l'architecture, de l'utilisation ou de l'usage d'un artefact et (2) d'engager des acteurs dans le processus de conception au-delà des rôles formels auxquels 
Vers un design accessible, au-delà du design de la forme et de la fonction

ils sont tenus (Zacklad, 2017, s.p.).

Ces deux spécificités sont également visibles dans les pratiques des concepteurs et donnent lieu à deux grandes catégories de savoirs. D'une part, le design de concepts, focalisé sur la production des caractéristiques de l'artefact à concevoir (objet, visuel, interaction, service, environnement). Zacklad distingue ici :

- le design de forme, qui concerne toutes les techniques de concrétisation de l'artefact à concevoir et qui relève de domaines tels que le graphisme, la typographie, la photo, la vidéo, etc. ;

- le design de fonction, qui s'intéresse à la définition du problème sans se compromettre dans les décisions liées aux technologies de mise en œuvre ;

- le design d'usage ou d'expérience, qui, à la différence du design de fonction focalisé sur l'« usabilité », aborde la problématique de l'usage et renvoie à une vision plus englobante des pratiques, aux environnements personnels et professionnels dans lesquels le nouvel artefact doit s'insérer ainsi qu'à la diversité des utilisateurs potentiels ;

- le design du symbolique, concerné par les aspects les plus immatériels et culturels des produits et services. Les dimensions fonctionnelles et expérientielles des artefacts tirent en partie leur effectivité du plan symbolique.

D'autre part, le design de relations, qui relève des pratiques d'animation du processus de conception et d'engagement des sujets, dans le cadre d'un agencement spatial et temporel adapté, et dans le but ultime de provoquer la créativité, la coopération inventive ainsi que l'élaboration des propositions. Zackald reconnaît ici :

- le design maïeutique ou design thinking, cherchant à générer des conditions de créativité, tout en tenant compte des contraintes spatiotemporelles, pour que les sujets puissent produire des nouvelles solutions de design ;

- le design participatif ou co-design, revendiquant dans sa démarche une forme de démocratie de la conception et 
d'émancipation des utilisateurs - légitimée par leur connaissance des situations réelles auxquelles les solutions de design sont destinées - ;

- et le design rhétorique, mettant en valeur les propositions des concepteurs lors de briefs destinés aux clients.

\section{Des règles d'accessibilité ancrées sur la forme et la fonction}

D'après la taxonomie du design présentée ci-dessus, nous montrerons que les principes directeurs des standards d'accessibilité sont principalement associés au design de la forme et de la fonction des interfaces numériques.

La norme française - le RGAA - repose sur des recommandations reconnues internationalement: les règles d'accessibilité pour les contenus web, ou WCAG 2.0 (pour l'anglais Web Content Accessibility Guidelines). Les WCAG 2.0 sont émises et actualisées par la Web Accessibility Initiative (WAI) du W3C, suivies par les praticiens du domaine et transposées en tant que norme ISO depuis 2012. Elles sont structurées selon quatre principes fondamentaux : perceptible, utilisable, compréhensible et robuste. Un contenu est perceptible s'il n'est pas invisible à tous les sens de l'usager ; il est utilisable s'il n'exige aucune interaction que l'usager ne peut réaliser ; il est compréhensible si l'usager est capable de saisir l'information ainsi que le fonctionnement de l'interface ${ }^{1}$; et il est robuste s'il peut être interprété de manière fiable par une large variété d'agents utilisateurs ${ }^{2}$, y compris les technologies d'assistance ${ }^{3}$ (W3C, 2014a).

\footnotetext{
${ }^{1}$ Les standards d'accessibilité utilisent le terme interface pour parler des éléments du contenu qui admettent une manipulation par l'usager, par exemple : un bouton, un lien, une liste déroulante, etc.

${ }^{2}$ Un agent utilisateur est, pour la WAI, un logiciel qui récupère et présente le contenu web aux utilisateurs, par exemple : les navigateurs web, les lecteurs multimédias, les modules d'extension, les technologies d'assistance, etc.

${ }^{3}$ Une technologie d'assistance est un matériel ou un logiciel qui fournit des fonctionnalités répondant aux besoins des utilisateurs ayant des limitations fonctionnelles. Elle peut notamment fournir des présentations de remplacement (comme de la synthèse vocale ou du contenu agrandi), des méthodes de saisie alternatives
} 
Vers un design accessible, au-delà du design de la forme et de la fonction

Pour chaque principe, on retrouve un ensemble de règles qui constituent les objectifs de base que les auteurs doivent atteindre. Par exemple, le principe de perceptibilité contient la règle "Faciliter la perception visuelle et auditive du contenu par l'utilisateur " (W3C et Association BrailleNet, 2009). Chaque règle comprend à la fois des critères de succès qui peuvent être testés sur une page web : des énoncés qui peuvent être qualifiés de vrais ou faux lorsqu'ils sont évalués sur un contenu spécifique. Ainsi, la règle suscitée inclut le critère suivant: "la couleur n'est pas utilisée comme la seule façon de véhiculer de l'information, d'indiquer une action, de solliciter une réponse ou de distinguer un élément visuel » (ibid., s. p.).

Le principe de perceptibilité est clairement associé au design de forme comme qu'il regroupe des critères de succès qui concernent les modes de concrétisation de l'artefact - ici de concrétisation de l'information numérique. Les recommandations pour la perceptibilité du contenu relèvent principalement du format de l'information (par ex.: tout contenu non textuel présenté à l'utilisateur a un équivalent textuel), de la mise en forme (par ex. : un rapport de contraste d'au moins 4,5:1 entre la couleur du texte et la couleur du fond), de la structure (par ex. : la structure et les relations véhiculées par le rendu d'un contenu peuvent être déterminées par un programme informatique), ainsi que de l'ordre (par ex.: lorsque l'ordre de présentation du contenu affecte sa signification, un ordre de lecture correct peut être déterminé par un programme informatique). II faut mentionner que les règles d'accessibilité s'intéressent à des formes de l'artefact autres que visuelles, notamment les contenus sonores de la page web.

Le principe d'utilisabilité est par définition associé au design de fonction. En effet, les critères de succès ramassés dans ce principe visent à faciliter l'utilisation de l'artefact en suggérant que toutes les fonctionnalités soient accessibles au clavier, que l'utilisateur ait suffisamment de temps pour lire et utiliser le contenu, que la page web soit exempte de tout élément susceptible de provoquer des crises et que des repères soient

(comme la voix), des mécanismes de navigation ou d'orientation supplémentaires et des transformations de contenu (comme rendre un tableau plus accessible). 
présents pour naviguer, trouver le contenu et se situer dans le site.

Le principe de compréhensibilité dédie une première règle à la lisibilité du contenu textuel - et donc au design de forme - qui stipule que la langue du texte doit être explicite, de même que la signification des mots rares et des abréviations, entre autres. Les deux autres règles du principe sont dédiées à la compréhension de l'utilisation de l'interface - et donc au design de fonction. La première traite de la prévisibilité du fonctionnement, à savoir que le contexte ne doit pas changer sans prévenir l'usager et que les mécanismes de navigation ainsi que l'identification des fonctionnalités doivent être cohérents dans l'ensemble du site web. La dernière règle vise à aider l'usager à éviter et à corriger les erreurs de saisie.

Enfin, le principe de robustesse veille à optimiser la compatibilité du contenu avec d'autres artefacts: les agents utilisateurs. C'est le design du programme informatique (le design du code source) qui va favoriser ou défavoriser cette compatibilité. Bien que tacite dans la théorie de Zacklad (2017), la conception de programmes informatiques correspond bien à un design de forme, à une élaboration du signifiant des signes performatifs (Bootz, 2006), qui reste cachée aux sens de l'utilisateur, mais de laquelle dépendent pourtant toutes les autres formes perceptibles de l'artefact.

\section{Les limites avouées des règles d'accessibilité}

La littérature scientifique qui aborde les limites des standards d'accessibilité signale principalement une lacune sur le plan du design d'usage ou d'expérience. Des recherches empiriques simulant des situations d'usage à travers des tests utilisateur (Power et al., 2012 ; Rømen et Svanæs, 2011) montrent que les WCAG couvrent à peine la moitié des problèmes rencontrés par les usagers en situation de handicap. Dans le même sens, mais depuis une approche de l'activité située, Gouédard, Folcher et Lompré (2012) ont étudié les usages d'une bibliothèque numérique par des personnes en situation de handicap et mis en évidence que les besoins essentiels relevés ne sont que partiellement 
Vers un design accessible, au-delà du design de la forme et de la fonction

couverts par les normes formelles.

Cooper, Kelly, Sloan et d'autres (Cooper et al., 2012 ; Kelly et al., 2009 ; Sloan et Kelly, 2011)soutiennent que l'accessibilité ne peut pas se réduire à la conformité d'une ressource numérique avec des directives techniques, mais qu'il faut adopter une approche relationnelle: l'accessibilité serait une propriété de la relation entre l'usager et la ressource dans le contexte où cette relation est médiée et non une propriété de la ressource en soi. Pour Di Blas, Paolini et Speroni (2004), les règles de la WAI peuvent garantir la lisibilité technique d'une page web - que la page soit lue par les technologies d'assistance -, mais sont incapables de fournir une expérience utilisateur effective.

Le caractère peu prescriptif des normes d'accessibilité en ce qui concerne le design de relation est un autre constat marquant. Même si la méthodologie standardisée pour évaluer la conformité d'un site web (W3C, 2014b) fait allusion à l'importance d'intégrer des personnes en situation de handicap dans le processus d'évaluation, elle le fait en tant que prérequis facultatif et n'émet aucune recommandation sur la manière d'engager les acteurs. II n'est pas hasardeux que les labellisations d'accessibilité fournies par des associations du monde du handicap, telles que le label Fédération des Aveugles et Amblyopes de France ${ }^{4}$, soient assez inhabituelles. La participation active des personnes en situation de handicap dans le processus d'élaboration des standards d'accessibilité est en soi un thème controversé (Adam et Kreps, 2009), même si l'équipe qui travaille actuellement pour la prochaine version des standards est fortement engagée dans ce sens (Lauriat et Spellman, 2017).

Par ailleurs, les standards d'accessibilité sont conçus et formulés pour accompagner un processus d'évaluation de l'existant. S'ils peuvent servir de guide dans le processus de conception, ils dénotent de vrais manques quant à la façon d'agencer les parties prenantes dans l'espace et dans le temps, de provoquer leur créativité, de les faire coopérer ou de les faire adhérer aux propositions de solution. Les travaux de recherche sur

${ }^{4}$ Voir : https://www.aveuglesdefrance.org/label-federation-des-aveugles-et-amblyopesde-france. 
le design de relation participatif sont également peu nombreux dans le champ de l'accessibilité (Brock et al., 2010 ; Folcher et Lompré, 2012 ; Laitano, 2017 ; Metatla et al., 2015).

Finalement, les réponses aux sondages sur les préférences des usagers d'un lecteur d'écran témoignent, à notre avis, de l'omission du design du symbolique dans les consignes d'accessibilité actuelles. Lorsque ceux-ci ont été interrogés sur les images du Web qui tentent de générer une certaine émotion ou du sentiment, $59 \%$ des 1121 répondants ont déclaré qu'ils préféraient que ces images soient décrites par leur lecteur d'écran, contre $31 \%$ qui ont souhaité que ces images soient ignorées et $10 \%$ qui n'avaient pas d'opinion (WebAIM, 2009). Plus encore, la vaste majorité de ceux qui ont répondu que ces images devaient être ignorées était des usagers qui se servent du lecteur d'écran pour évaluer l'accessibilité, mais qui ne se trouvent pas en situation de handicap visuel. Ce résultat est clairement opposé au critère de succès 1.1.1 des WCAG 2.0, selon lequel les images décoratives ne doivent pas être décrites par les technologies d'assistance.

\section{Pistes pour un design accessible du symbolique}

Comme le rappelle l'approche radicale du design (section 2), le design du symbolique - celui qui relève de la signification des interfaces - joue un rôle fondamental, dont les dimensions fonctionnelles et expérientielles tirent aussi parti. Pourtant, il est davantage absent dans la littérature du design accessible. À partir de notions communicationnelles qui ont été réinvesties dans le numérique, cette dernière section ouvre quelques pistes pour développer un design accessible du symbolique. Elles sont illustrées sur le site web de l'Assurance Maladie de Paris ${ }^{5}$ concerné par la loi française d'accessibilité numérique, mais qui déclare ne pas valider à présent tous les critères attendus.

Les notions de genre et de finalité communicationnelle - le but que le concepteur de l'interface poursuit vis-à-vis des usagers (Crozat et al., 2012) - sont fréquemment transposées à la communication numérique (ibid.). Tel est le cas des genres du

${ }^{5}$ Voir : https://www.ameli.fr/paris (consulté le 4 janvier 2018). 
Vers un design accessible, au-delà du design de la forme et de la fonction

Web (Lindemann et Littig, 2010) (blogue, communautaire, institutionnel, informatif, boutique, académique...), qui peuvent poursuivre des finalités communicationnelles bien différentes (descriptive, narrative, argumentative, persuasive, incitative...). La section d'actualités pour les assurés ${ }^{6}$ du site ameli.fr reproduit, par exemple, un genre informatif simplifié, caractérisé par un ensemble de billets ordonnés temporellement par leur date de parution. Les billets sont toujours composés de texte, d'hyperliens et, éventuellement, d'images et de vidéos.

Regardons d'abord les images, définies par les WCAG 2.0 comme un contenu non textuel pour lequel le concepteur doit fournir un équivalent textuel ${ }^{7}$ remplissant la même fonction (W3C et Association BrailleNet, 2009). Dans sa classification des types d'images (Leiserson et McGee, 2015), la WAl distingue: des images informatives, telles que les photographies et les illustrations; des images de décoration visuelle; des images fonctionnelles, telles que les icônes qui fonctionnent comme des boutons ; des images de texte; des images complexes, telles que les graphiques et les diagrammes; des groupes d'images; et des cartes d'images. Cette typologie des images est, comme nous l'avons déjà signalé, fondée principalement sur le design de forme.

Les images des billets de la rubrique "Actualités " seraient, du point de vue des standards, des images informatives devant porter pour équivalent une courte description qui véhicule les informations essentielles présentées par l'image. Ainsi, pour la photographie du billet « Le diabète : comment l'éviter ? » (Figure 1), les standards d'accessibilité pourraient admettre un équivalent du type : " Deux femmes roulent en vélo dans la campagne ${ }^{8}$ ". Or, replacée dans son genre informatif à finalité clairement préventive - agir contre le diabète -, la photographie gagnerait à être traduite comme «Faire du vélo régulièrement contribue à prévenir le diabète ". Cet équivalent, exhortatif et non pas descriptif, serait davantage pertinent en vue de la finalité communicationnelle.

\footnotetext{
${ }^{6}$ Voir : https://www.ameli.fr/paris/assure/actualites (consulté le 4 janvier 2018).

${ }^{7}$ Un équivalent textuel est un texte associé par programmation à l'image, notamment à partir de l'attribut ALT en HTML.

${ }^{8}$ À noter que l'équivalent actuel de la photographie, à savoir « image_diabete », est inaccessible même du point de vue des WCAG 2.0.
} 


\section{María Inés LAITANO}

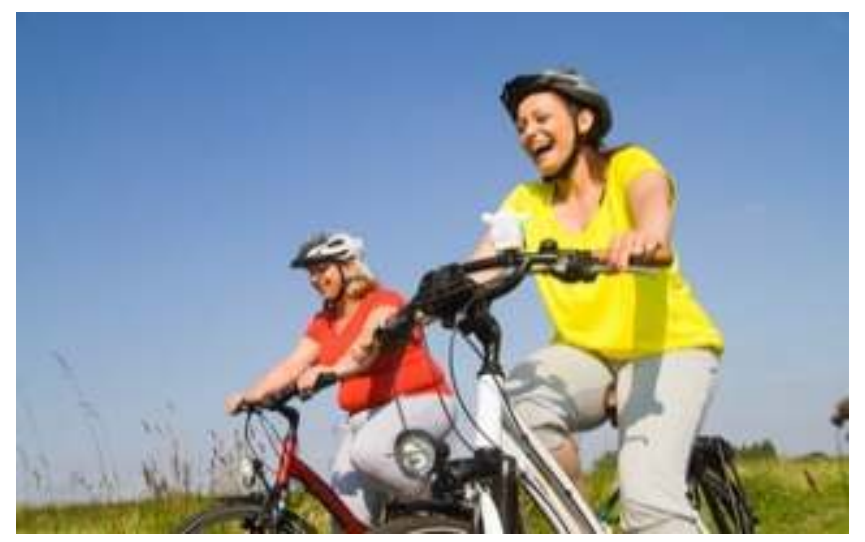

Figure 1. Photographie accompagnant le billet à propos de la prévention du diabète sur le site de l'Assurance Maladie

(Source : https://www.ameli.fr/paris/assure/actualites/le-diabete-comment-leviter)

II en va de même pour l'accessibilité des contenus vidéo: les WCAG 2.0 exigent qu'une audiodescription ou une version de remplacement descriptive ${ }^{9}$ accompagne les médias temporels, ignorant ainsi les finalités communicationnelles qui visent un effet autre que la description. Une version de remplacement pour la vidéo " Mal de dos ? Le bon traitement, c'est le mouvement ${ }^{10}$ ", du point de vue des standards, aurait tendance à décrire textuellement l'effet visuel de fusion entre le dos des personnages et les différentes activités physiques. La vidéo fait cependant partie d'une campagne de sensibilisation qui a l'intention de persuader par cet effet vif et immédiat. Une version de remplacement sonore qui intègre notamment des icônes auditives (Gaver, 1989) évoquant les différentes activités physiques serait susceptible de créer le même effet pour un public n'ayant pas accès au visuel.

\footnotetext{
${ }^{9}$ Une version de remplacement est un document contenant, dans un ordre correct, une description des contenus visuels et sonores de la vidéo.

${ }^{10}$ Voir : https://www.youtube.com/watch?v=9MDIwhmU2IA.
} 
Vers un design accessible, au-delà du design de la forme et de la fonction

En tant que composante essentielle du Web, l'hyperlien fait l'objet des recommandations d'accessibilité. Les WCAG 2.0 établissent que le texte de l'hyperlien doit décrire la fonction de celui-ci - la fonction étant ici comprise comme la nature du résultat obtenu par l'activation de l'hyperlien (W3C et Association BrailleNet, 2009). La compréhension de l'hyperlien est particulièrement importante pour les usagers d'un lecteur d'écran qui sont parfois confrontés à la lecture du lien en dehors du texte environnant. Bien que décrire la nature du document relié par un hyperlien puisse donner lieu à des dénominations fortement divergentes, la WAI ne fait pas de suggestions particulières sur cette tâche de rédaction. Comme précédemment, notre proposition est de composer le texte de l'hyperlien en fonction de la finalité communicationnelle, en nous inspirant librement des potentiels expressifs de l'hyperlien tels qu'ils sont abordés par Saemmer (2015).

Par l'analyse d'hypertextes dans la presse en ligne et la littérature numérique, Saemmer (2015) pose des registres discursifs dans lesquels l'hyperlien aurait tendance à s'inscrire: l'informationnel, lorsque l'hyperlien fait de "simples " renvois vers des définitions, des informations contextuelles ou autres ; l'argumentatif, lorsqu'il sert à augmenter, à expliquer ou à dialectiser une argumentation principale (celle du document géniteur); et le registre de la figure de rhétorique, lorsqu'il ironise ou subvertit les propos du document géniteur. Des sous-catégories sont ensuite avancées, notamment dans les hypertextes argumentatifs en associant fonction de l'hyperlien et connecteur d'argumentation : autorité, analogie, amplification de l'argument de base, etc.

Pour nous, énoncer un hyperlien de manière accessible reviendrait à tenir compte, dès le texte de l'hyperlien, des effets de sens prévus par le concepteur. Cela nuance, voire complète, la règle d'accessibilité, car il n'est pas uniquement question de faire explicite ou de décrire la nature du document relié - ce n'est d'ailleurs pas le cas dans les hyperliens rhétoriques -, mais aussi d'introduire ou d'anticiper une intention communicationnelle. Prenons pour illustration le billet "L'aide au paiement d'une 
complémentaire santé, un droit méconnu ${ }^{11} "$, qui présente des hyperliens informationnels, argumentatifs, mais aussi des hyperliens de type incitatif ${ }^{12}$ qui encouragent ici les assurés à faire valoir un droit. Dans cette dernière catégorie, nous retrouvons, par exemple, un hyperlien dénommé "un simulateur », qui renvoie vers le simulateur pour estimer ses droits. Une expression qui inciterait davantage l'usager, en manifestant de manière explicite l'invitation à cliquer, serait: "Estimez en quelques clics vos droits à une aide pour une complémentaire santé ». Parmi les hyperliens à fonction argumentative, un lien sur l'expression "depuis le $1^{\text {er }}$ octobre 2017 » renvoie vers un autre billet qui confirme le plafonnement des tarifs de certains soins dentaires. Au lieu d'énoncer uniquement la date, l'hyperlien gagnerait à synthétiser l'argument qui est développé dans le texte relié (par exemple: «certains tarifs de soins dentaires sont plafonnés depuis octobre 2017 »). Enfin, les hyperliens informatifs devraient davantage préciser la source d'information à laquelle ils renvoient, par exemple utiliser l'expression "le site d'information de l'ACS " au lieu de « info-acs.fr».

\section{Conclusion}

Tout comme d'autres législations dans le monde, la loi française reconnaît les bénéfices potentiels de l'accessibilité numérique pour l'inclusion sociale et incite les services publics à respecter les standards internationaux d'accessibilité. II est cependant avoué qu'une communication numérique pleinement accessible n'est pas garantie par la seule conformité aux règles de la W3C. En nous appuyant sur la typologie des savoirs du design proposée par Zacklad (2017), nous avons développé en quoi les normes sont principalement axées sur le design de la forme et de la fonction des interfaces numériques, au détriment du design du symbolique, de l'usage et des relations. En effet, se consacrant à la perceptibilité, à l'utilisabilité, à la compréhensibilité et à la

\footnotetext{
${ }^{11}$ https://www.ameli.fr/paris/assure/actualites/laide-au-paiement-dune-complementairesante-un-droit-meconnu (consulté le 4 janvier 2018)

${ }^{12}$ Certainement ce registre discursif n'est pas identifié par Saemmer car elle ne traite pas d'hypertextes à fonction de service dans ces corpus d'analyse.
} 
Vers un design accessible, au-delà du design de la forme et de la fonction

robustesse du contenu, les règles d'accessibilité ne font que regarder la matérialité et l'utilité de l'artefact à concevoir. Des travaux issus du champ du design accessible confirment l'insuffisance des standards et recommandent de concevoir une expérience utilisateur accessible.

Nous avons exploré la voie du design accessible du symbolique, moins traitée dans la littérature, en proposant et en illustrant quelques pistes de réflexion à partir de concepts communicationnels appliqués au numérique. Aborder le design de l'interface par son genre et sa finalité communicationnelle donnerait lieu à des solutions accessibles qui vont au-delà de la reproduction "automatique " d'un contenu dans une autre modalité. La mise en accessibilité d'images, de contenu vidéo et d'hyperliens est une parmi d'autres préoccupations pertinentes pour le design du symbolique. Ainsi, la communicabilité de l'interface que de Souza (2013) définit comme sa capacité à signifier et à communiquer l'intention des concepteurs pourrait-elle devenir demain le cinquième principe des règles d'accessibilité.

Les carences des standards d'accessibilité sur le plan du design du symbolique, de l'usage et des relations font appel à de nouvelles recherches en SIC et en design, tout aussi intéressantes pour la formation, pour les acteurs publics concernés ainsi que pour ceux chargés d'élaborer de futures normes d'accessibilité.

\section{Références}

Adam, A. et Kreps, D. (2009). Disability and discourses of web accessibility. Information, Communication et Society, 12(7), 1041-1058. doi: 10.1080/13691180802552940

Balin, P. et Gossart, C. (2015). L'accessibilité des TIC par les personnes handicapées : état des lieux du contexte actuel. Terminal. Technologie de l'information, culture et société, (116). doi: 10.4000/terminal.751

Bootz, P. (2006). Digital Poetry: From Cybertext to Programmed Forms. Leonardo Electronic Almanac, 14(5). Repéré à http://leoalmanac.org/journal/vol_14/lea_v14_n05-06/pbootz.asp 
BrailleNet (2014). Ce que les sites Web publics nous disent de leur accessibilité. Repéré à

http://www.braillenet.org/documents/communique-braillenet2014-03-25.pdf

Brock, A., Vinot, J.-L., Oriola, B., Kammoun, S., Truillet, P. et Jouffrais, C. (2010). Méthodes et outils de conception participative avec des utilisateurs non-voyants. Proceedings of the $22^{\text {nd }}$ Conference on l'Interaction Homme-Machine (p. 65-72). New York, NY : ACM Press.

doi: 10.1145/1941007.1941017

Cooper, M., Sloan, D., Kelly, B. et Lewthwaite, S. (2012). A Challenge to Web Accessibility Metrics and Guidelines: Putting People and Processes First. Dans Proceedings of the International Cross-Disciplinary Conference on Web Accessibility (p. 201-204). New York, NY : ACM. doi: 10.1145/2207016.2207028

Crozat, S., Bachimont, B., Cailleau, I., Bouchardon, S. et Gaillard, L. (2012). Éléments pour une théorie opérationnelle de l'écriture numérique. Document numérique, 14(3), 9-33.

de Souza, C. S. (2013). Semiotics. Dans A. Zahirovic et al. (dir.), The Encyclopedia of Human-Computer Interaction ( $2^{\mathrm{e}}$ éd.).

Aarhus, Danemark : The Interaction Design Foundation. Repéré à http://www. interactiondesign.org/encyclopedia/semiotics_and_humancomputer interaction.html

Di Blas, N., Paolini, P. et Speroni, M. (2004). "Usable accessibility" to the Web for blind users. Proceedings of $8^{\text {th }}$ ERCIM Workshop “User Interfaces for All" (p. 109-126). Viennes. Repéré à http://www.ui4all.gr/workshop2004/files/ui4all_proceedings/adju nct/accessibility/109.pdf

Folcher, V. et Lompré, N. (2012). Accessibilité pour et dans l'usage : concevoir des situations d'activité adaptées à tous et à chacun. Le travail humain, 75(1), 89-120.

Gaver, W. W. (1989). The SonicFinder: An interface that uses auditory icons. Human-Computer Interaction, 4, 67-94. 
Vers un design accessible, au-delà du design de la forme et de la fonction

Gouédard, C., Folcher, V. et Lompré, N. (2012). Une bibliothèque numérique à l'épreuve de la déficience : études de cas.

Activités, 9(1). doi: 10.4000/activites.170

Kelly, B., Sloan, D., Brown, S., Seale, J., Lauke, P., Ball, S. et Smith, S. (2009). Accessibility 2.0: Next Steps for Web Accessibility. Journal of Access Services, 6(1-2), 265-294. doi: 10.1080/15367960802301028

Laitano, M. I. (2017). Developing a Participatory Approach to Accessible Design. International Journal of Sociotechnology and Knowledge Development (IJSKD), 9(4), 1-11. doi: 10.4018/IJSKD.2017100101

Lauriat, S. et Spellman, J. (2017, mars). What Comes After WCAG 2.1?. Communication présentée à CSUN 2017 Assistive Technology Conference. Repéré à goo.gl/3SK8T9

Leiserson, A. B. et McGee, L. (2015). Informative Images. Repéré à https://www.w3.org/WAl/tutorials/images/informative/

Lespinet-Najib, V., Pinède, N., Belio, C., Demontoux, F. et Liquète, V. (2015). L'accessibilité Web, en 2013, en France : enquête nationale sur les pratiques et les usages des professionnels du Web. Terminal. Technologie de l'information, culture et société, (116). doi: $10.4000 /$ terminal.649

Lindemann, C. et Littig, L. (2010). Classification of Web Sites at Super-genre Level. Dans A. Mehler, S. Sharoff et M. Santini (dir.), Genres on the Web (p. 211-235). New York, NY : Springer Netherlands. doi: 10.1007/978-90-481-9178-9_10

Metatla, O., Bryan-Kinns, N., Stockman, T. et Martin, F. (2015). Designing with and for people living with visual impairments: Audio-tactile mock-ups, audio diaries and participatory prototyping. CoDesign, 11(1), 35-48. doi: 10.1080/15710882.2015.1007877

Power, C., Freire, A., Petrie, H. et Swallow, D. (2012). Guidelines are only half of the story: Accessibility problems encountered by blind users on the Web. Proceedings of the SIGCHI Conference on Human Factors in Computing Systems (p. 433-442).

New York, NY : ACM. doi: 10.1145/2207676.2207736 
Rømen, D. et Svanæs, D. (2011). Validating WCAG versions 1.0 and 2.0 through usability testing with disabled users. Universal Access in the Information Society, 11(4), 375-385. doi: 10.1007/s10209-011-0259-3

Saemmer, A. (2015). Rhétorique du texte numérique : figures de la lecture, anticipations de pratiques. Villeurbanne, France : Presses de l'Enssib.

SGMAP (France). (2017). Référentiel Général d'Accessibilité pour les Administrations (RGAA) Version 3 2017. Repéré à https://references.modernisation.gouv.fr/rgaa-accessibilite/

Sloan, D. et Kelly, B. (2011). Web accessibility metrics for a post digital world. Website Accessibility Metrics symposium. Repéré à http://www.w3.org/WAI/RD/2011/metrics/paper10/

W3C. (2014a). Understanding WCAG 2.0. Repéré à http://www.w3.org/TR/UNDERSTANDING-WCAG20/

W3C. (2014b). Website Accessibility Conformance Evaluation Methodology (WCAG-EM) 1.0. Repéré à http://www.w3.org/TR/WCAG-EM/

W3C et Association BrailleNet. (2009). Règles pour l'accessibilités des contenus Web (WCAG) 2.0. Repéré à http://www.w3.org/Translations/WCAG20-fr/

WebAIM. (2009, octobre). Screen Reader User Survey \#2 Results. Repéré à http://webaim.org/projects/screenreadersurvey2/\#images

Zacklad, M. (2017). Design et création : vers une théorie interdisciplinaire radicale du Design. Wikicréation. Repéré à http://www.wikicreation.fr/fr/ 